Revista Tecné, Episteme y Didaxis: TED. Año 2014, Número Extraordinario. ISSN Impreso: 0121-3814, ISSN web: 2323-0126

Memorias, Sexto Congreso Internacional sobre Formación de Profesores de Ciencias. 08 al 10 de octubre de 2014, Bogotá

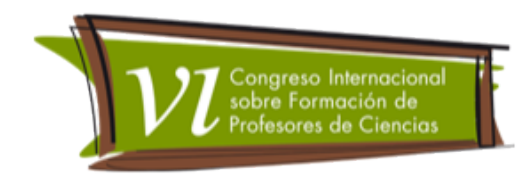

\title{
La enseñanza del concepto de fem en el contexto de la inducción electromagnética
}

Oyuela, Diana'; Garzón Isabel² y Zambrano, Tufik $^{3}$

Categoría 2. Trabajo de investigación

\section{Resumen}

En el presente trabajo se describe la monografía titulada "La enseñanza del concepto de fem en el contexto de la inducción electromagnética", en la cual se desarrolló una secuencia de enseñanza; con el propósito de presentar una alternativa para enseñar el concepto de fem inducida, respaldada en un estudio teórico entorno al concepto y una reflexión acerca de los elementos que componen el proceso de enseñanza-aprendizaje; hecha a través del modelo de aprendizaje como investigación orientada ( $\mathrm{MAClO}$ ). Finalmente, se expone una conclusión que expresa la importancia que tiene este tipo de diseños para un profesor en formación.

\section{Palabras clave}

Fuerza electromotriz, inducción electromagnética, observador inercial, secuencia de enseñanza, MACIO.

\section{Abstract}

The present paper describes the monograph entitled "Teaching the concept of emf in the context of electromagnetic induction," in this document a sequence of teaching was developed; which presents an alternative to teach the concept of induced emf, backed by a theoretical study around the concept and a reflection on the elements of the teaching-learning process; made through the learning model as oriented research. finally, a conclusion expresses the importance of this type of design for a trainee teacher.

\footnotetext{
${ }^{1}$ Universidad Pedagógica Nacional, diana.oyvela@gmail.com

2 Universidad Pedagógica Nacional, igarzon@pedagogica.edu.co

3 Universidad Pedagógica Nacional, tzambrano@pedagogica.edu.co
} 
Revista Tecné, Episteme y Didaxis: TED. Año 2014, Número Extraordinario. ISSN Impreso: 0121-3814, ISSN web: 2323-0126

Memorias, Sexto Congreso Internacional sobre Formación de Profesores de Ciencias. 08 al 10 de octubre de 2014, Bogotá

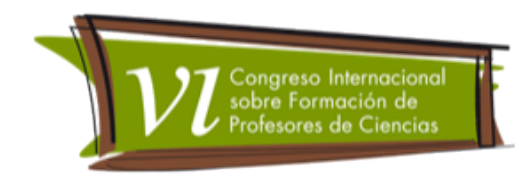

\section{Keywords}

Electromotive force, electromagnetic induction, inertial observer, teaching sequence, Learning model as oriented research.

\section{Objetivos}

El problema que configura la investigación presente en este trabajo radica en investigar ¿ Cómo abordar la enseñanza del concepto de fem en el marco del fenómeno de inducción electromagnética? Esta cuestión se resuelve a través del cumplimiento de los siguientes objetivos:

- Realizar un análisis conceptual de la fuerza electromotriz en el marco del fenómeno de inducción electromagnética, considerando diferentes observadores inerciales.

- Elaborar una secuencia de enseñanza para explicar el concepto de fem en el contexto de la inducción electromagnética, haciendo uso del modelo de aprendizaje como investigación orientada.

- Analizar los planteamientos del Modelo de Aprendizaje Como Investigación Orientada y sus aplicaciones en la secuencia de enseñanza

\section{Marco teórico}

Este trabajo se ve comprometido, en primer lugar, a potenciar el interés de los estudiantes por aprender el concepto de fem y, en segundo lugar, a propiciar en los estudiantes una concepción de este lo más cercana posible a la aceptada científicamente. Para lograr lo anterior este proyecto hará uso del modelo de aprendizaje como investigación orientada, para plantear una secuencia de enseñanza en la que se pone en práctica la afirmación de Bacherlard (Citada en el libro de la UNESCO) "todo conocimiento es la respuesta a una cuestión".

Cabe resaltar que la fuerza electromotriz es un concepto que pertenece a la Teoría Electromagnética de Campos (TEC) y que se puede abordar en diferentes contenidos de un curso de introducción al electromagnetismo. En la monografía descrita en este artículo, se estudia el concepto de fem desde el fenómeno de inducción electromagnética teniendo en cuenta tres aspectos fundamentales: 1) Mostrar la fem como un concepto único para todos los contextos de la TEC, es decir hacer énfasis en que la fem es solo una y no existen clases de ella; 2) Mostrar dos modos diferentes de generar una fem en el contexto del fenómeno de inducción electromagnética (por el cambio en la intensidad de un campo magnético o por el movimiento de un conductor en un campo magnético 
Revista Tecné, Episteme y Didaxis: TED. Año 2014, Número Extraordinario. ISSN Impreso: 0121-3814, ISSN web: 2323-0126

Memorias, Sexto Congreso Internacional sobre Formación de Profesores de Ciencias. 08 al 10 de octubre de 2014, Bogotá

constante) y 3) Mostrar el carácter relativo de los dos modos de generar la fem inducida (por esta razón se hace uso de observadores inerciales para describir el fenómeno de inducción electromagnética).

\section{Metodología}

La primera parte del trabajo esbozado en la monografía que se describe en este artículo, radica en una investigación documental, en la cual se recopiló información sobre el concepto de fem, los modos de generar la fem y la descripción de fenómenos de inducción electromagnética utilizando diferentes observadores inerciales; en el desarrollo de esta tarea se determinaron los elementos necesarios de un aprendizaje significativo del concepto de fem, que posteriormente se plasmaron en los indicadores de aprendizaje. Paralelamente se realizó una investigación acerca del $\mathrm{MAClO}$, del cual se analizaron planteamientos que consecutivamente se aplicaron para diseñar la secuencia de enseñanza.

\section{Resultados}

Con el fin de cumplir el propósito de abordar la enseñanza del concepto de fem en el contexto de la inducción electromagnética, el primer objetivo que se conquistó radica en un estudio teórico que se puede resumir en los hallazgos más importantes, como por ejemplo, la definición de fem útil para toda la teoría electromagnética, la caracterización de la fem inducida y el carácter relativo de la fem inducida.

Así, para exponer uno de los resultados del análisis conceptual, de manera general se pude afirmar que para la teoría electromagnética de campos, la fem es una magnitud que cuantifica una transferencia de energía por unidad de carga asociada a un campo no conservativo, definido para la acción no eléctrica y no conservativa total por unidad de carga de prueba. Es conveniente resaltar que en cada caso donde el término fem es usado, constituye una propiedad de los generadores de energía eléctrica y por ende una medida de la energía que el generador suministra a una unidad de carga.

Con relación al cumplimiento del segundo objetivo; se presentan en la tabla 1, a modo de ejemplo, algunas de las actividades y preguntas que componen la secuencia de enseñanza en las que se ve reflejada la definición de fem inducida hecha en el análisis conceptual, por su utilización en los generadores. Para señalar las preguntas que orientan el aprendizaje se hace uso de la letra $P$, para 
Revista Tecné, Episteme y Didaxis: TED. Año 2014, Número Extraordinario. ISSN Impreso: 0121-3814, ISSN web: 2323-0126

Memorias, Sexto Congreso Internacional sobre Formación de Profesores de Ciencias. 08 al 10 de octubre de 2014, Bogotá

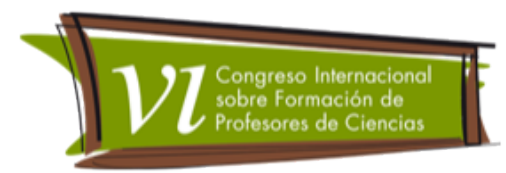

señalar las actividades se utiliza la letra A y para señalar las cuestiones que incentivan la autoevaluación de los estudiantes se emplea la letra C.

Tabla 1. Parte inicial de la secuencia de enseñanza

\section{LA PREGUNTA DE INVESTIGACIÓN PARA LOS ESTUDIANTES ES: ¿CÓMO ENCENDER UN DIODO HACIENDO USO DE IMANES?}

P.x ¿̇Qué importancia tiene investigar cómo encender un diodo haciendo uso de imanes?

${ }_{\boldsymbol{P} .2} \quad \dot{¿}$ De dónde proviene la energía eléctrica necesaria para encender las bombillas de su casa?

\section{Diseño de un circuito}

A.1 Construya el esquema de un circuito por el que circule corriente con el propósito de encender un diodo usando imanes. Puede cambiar los imanes por otra fuente de campo magnético.

P.s ¿¿Qué papel desempeñan los imanes en este circuito?

P.4 ¿ ¿Cuál es la fuente de energía eléctrica?

C1: ¿̇Logré construir hipótesis acerca del diseño del circuito?

\section{Generador eléctrico}

A.2 Un generador eléctrico es un dispositivo destinado para transformar energía mecánica en energía eléctrica, siga las indicaciones para construir un generador eléctrico casero usando los siguientes materiales.

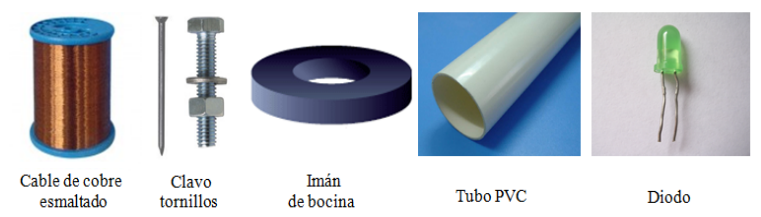

Figura 1 Materiales para construir un generador eléctrico

Para construir el generador es necesario fabricar un embobinado con núcleo de hierro, para esto se enrolla el cable de cobre en el clavo o tornillo como se evidencia en la figura 2(a), y soldar el diodo a los extremos del cable.

Coloque los imanes con los polos opuestos haciendo pasar el tubo PVC por el centro de ellos teniendo en cuenta que el diámetro de los imanes sea el adecuado para que uno quede fijo en la parte inferior del tubo y el otro se deslice a través del tubo, como muestra la figura 2(b), de esta manera queda armada la parte externa del generador. Ponga el embobinado en el interior del tubo y deslice el imán.

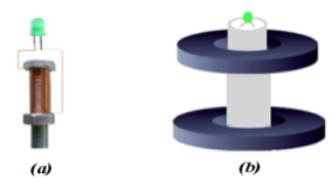


Revista Tecné, Episteme y Didaxis: TED. Año 2014, Número Extraordinario. ISSN Impreso: 0121-3814, ISSN web: 2323-0126

Memorias, Sexto Congreso Internacional sobre Formación de Profesores de Ciencias. 08 al 10 de octubre de 2014, Bogotá

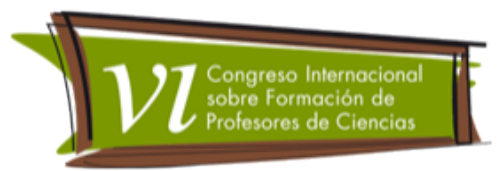

Figura 2 (a) Embobinado (b) Generador eléctrico casero

P.5 ¿ ¿Cuáles son las condiciones necesarias para que al deslizar el imán el diodo se encienda?

P.6 ¿ ¿Cómo explicaría el funcionamiento del generador eléctrico construido?

P.j ¿̇Qué magnitudes físicas puede usar para describir el funcionamiento del generador? ¿̇Cuáles magnitudes actúan como constantes y cuáles como variables?

p.s ¿ Con cuál magnitud física se puede medir la energía invertida para encender el diodo? Justifique su respuesta.

P.g ¿Qué ventajas tiene para el medio ambiente construir dispositivos tecnológicos alimentados de otras fuentes diferentes a las baterías?

C2: ¿̇obtuve una explicación del generador eléctrico a modo de hipótesis?

C3: ¿Desarrolle un análisis sobre las magnitudes físicas que intervienen en el generador eléctrico y determine cuál tiene relación con la medida de la energía?

Respecto al tercer objetivo, se hace necesario ejemplificar uno de los planteamientos del MACIO y su uso en la secuencia de enseñanza, para esto se explica la situación problemática de interés desde la que se desarrolló toda la secuencia de enseñanza, puesto que el MAClO propone iniciar el estudio de un tema bajo la idea de que todo conocimiento es la respuesta a una cuestión o a un problema (Bachelard, 1938)

En los planteamientos del $\mathrm{MAClO}$, la situación problemática de interés debe ser una situación con la que cualquier alumno pueda encontrarse frecuentemente o tener la capacidad de familiarizarse muy rápido. Para esto es necesario tener en cuenta el nivel de dificultad correspondiente a la zona de desarrollo próximo de los estudiantes a los que va dirigida la problemática (Vigotsky, 1988) y procurar que la situación exija un tratamiento inicial fundamentalmente cualitativo.

Tabla 2. Situación problemática de interés de la secuencia de enseñanza

¿Cómo encender un diodo haciendo uso de imanes? Es la situación problemática escogida para abordar el estudio del concepto de fem en la inducción electromagnética, la intención es plantear una problemática con el fin de que los estudiantes perciban la generación de energía eléctrica como un reto fundamental para la sociedad y como una tarea que merece pertenecer al desarrollo de la ciencia.

Esta pregunta generaliza los tópicos de la secuencia de enseñanza y tiene un enunciado que exige a los estudiantes disponer de una estrategia experimental apoyada de argumentos teóricos que les permita justificar la forma de proceder para dar una solución. El nivel de dificultad de la situación es adecuado porque los alumnos antes de estudiar la inducción electromagnética han investigado acerca de temas como los circuitos de corriente continua, las fuentes de campo magnético, el flujo de campo magnético y la fuerza de Lorentz; útiles para dar solución a la problemática planteada. 
Revista Tecné, Episteme y Didaxis: TED. Año 2014, Número Extraordinario. ISSN Impreso: 0121-3814, ISSN web: 2323-0126

Memorias, Sexto Congreso Internacional sobre Formación de Profesores de Ciencias. 08 al 10 de octubre de 2014, Bogotá

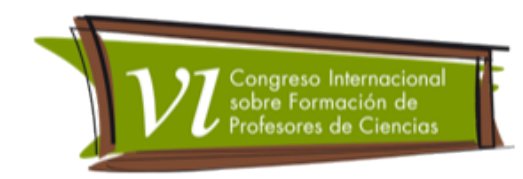

\section{Conclusiones}

Generalmente quien sabe mucho respecto a una disciplina es un apasionado por ella, sin embargo, no siempre esa pasión disciplinar está acompañada por un compromiso con el aprendizaje de otras personas. En esta dirección, se considera que el buen docente no es aquel que se preocupa únicamente por saber todo acerca de su disciplina, por el contrario, el buen docente es aquel que logra integrar sus conocimientos científicos y pedagógicos, sus ideologías y su experiencia para planificar y desarrollar la enseñanza. Por lo tanto, en esta monografía no solo se estudió el concepto de fem, también se lleva a cabo una reflexión acerca de los procesos de enseñanza y aprendizaje basada en el $\mathrm{MACIO}$.

EI MACIO funcionó como una filosofía del aprendizaje en la cual se proyectó la elaboración de la secuencia de enseñanza, pues un Modelo de aprendizaje es un plan estructurado que configura un estilo singular de aprendizaje esbozado desde perspectivas particulares de los elementos y sujetos que componen los procesos de enseñanza y aprendizaje. Sin embargo, con este trabajo se puede concluir que la efectividad de un modelo de enseñanza no radica únicamente en los resultados de implementar los diseños de enseñanza basados en el modelo, la efectividad del modelo se puede evidenciar, también, en los elementos que este aporte para que el profesor tenga las mejores herramientas a la hora de prestar su servicio como docente.

El logro con el que se finalizó la investigación se concreta en la estructura de la secuencia de enseñanza que no se resume en un conjunto de tareas a realizar, por el contrario es un proyecto de enseñanza diseñado con el fin de lograr el cumplimiento de los indicadores de aprendizaje y el cumplimiento de los objetivos que proponen los planteamientos del MAClO. La secuencia de enseñanza no se implementó, sin embargo, se establece como una alternativa para presentar el concepto de fem en el contexto de la inducción electromagnética. No obstante, en esta área la conclusión más significativa radica en resaltar la elaboración de un programa de enseñanza como uno de los procesos más importantes en la educación de los profesores en formación, debido a que este tipo de procesos permite reflexionar acerca del oficio docente y en este la importancia que tiene programar la enseñanza 
Revista Tecné, Episteme y Didaxis: TED. Año 2014, Número

Extraordinario. ISSN Impreso: 0121-3814, ISSN web: 2323-0126

Memorias, Sexto Congreso Internacional sobre Formación de Profesores de Ciencias. 08 al 10 de octubre de 2014, Bogotá

\section{Referencias biblográficos}

Bachelard, G. (1938). La formacion del espiritu cientifico. Mexico: Siglo XXI.

Galili, I; Kaplan, D; Lehavi, Y. (2006). Teaching Faraday's law of electromagnetic induction in an introductory physics course (Vol. 74). American Journal of Physics.

Guisasla, J., Montero, A., \& Fernández, M. (2008). La historia del concepto de fuerza electromotriz en circuitos eléctricos y la elección de indicadores de aprendizaje comprensivo. Revista Brasileira de Ensino de Física, 30(1),1604-1 a 1604-8.

Guisasola, J., Zubimendi, J., Almudi, J., \& Ceberio, M. (2007). Propuesta de enseñanza en cursos introductorios de física en la universidad, basada en la investigación didáctica: siete años de experiencia y resultados. Enseñanza de las ciencias, 25(1, 91-105).

Guisasola, J; Almudi, J; Zuza, K. (2010). Dificultades de los estudiantes universitarios en el aprendizaje de la inducción electromagnética. Enseñanza de las ciencias, 26(2), 1401-1 a 1401-9.

Guisasola, J; Montero, A; Fernández, M. (2005). Concepciones de futuros profesores de ciencias sobre un concepto olvidado en la enseñanza de la electricidad: La fuerza electromotriz. Enseñanza de las ciencias 23(1), 47-60.

Montero, A. (2007). El concepto de fuerza electromotriz en la interacción de circuitos de corriente estacionaria. Análisis critico de su enseñanza y propuesta didáctica alternativa. Editorial de la Universidad de Granada.

UNESCO. (2005). ¿Cómo promover el interés por la cultura científica? una propuesta didáctica fundamentada para la educación científica de jóvenes de 15 a 18 años. Publicado por la Oficina Regional de Educación de la UNESCO para América Latina y el Caribe.

Vigotsky, L. (1988). El desarrollo de los procesos psicológicos superiores. México: Crítica, Grupo Editorial Grijalbo. 